\title{
LIGHT-INDUCED GRATINGS IN CdMnTeSe:In CRYSTALS
}

\author{
B. Koziarska-Glinka ${ }^{a}$, M. Ponder $a$, T. Wojtowicz $a$, I. MiotKowski ${ }^{b}$, \\ J.M. LANGER ${ }^{a}$ AND A. SUCHOCKI ${ }^{a}$ \\ ${ }^{a}$ Institute of Physics, Polish Academy of Sciences \\ Al. Lotników 32/46, 02-668 Warsaw, Poland \\ ${ }^{b}$ Purdue University, West Lafayette, Indiana, 46 566, USA
}

\begin{abstract}
We show that DX-like centers in $\mathrm{Cd}_{1-x} \mathrm{Mn}_{x} \mathrm{Te}_{1-y} \mathrm{Se}_{x}$ :In crystal can be used in writing volume lolographic gratings. The scattering efficiency is higher than $10 \%$ which proves the dispersive character of the light-induced gratings. Two different metastable centers with different lattice relaxation were found in the crystal. Measurements of the power dependence of the degenerate four-wave mixing scattering efficiency testify that both of these centers have negative $U$ properties.
\end{abstract}

PACS numbers: 74.62.Dh, 61.72.Ji, 42.65.Hw

Recent interest in bistable dopants in semiconductors (and also in more ionic hosts as in $\mathrm{CdF}_{2}$ crystals) has been also motivated by potential applications of such materials for information storage [1-3]. Bistability means here electron trapping either at the localized, ground state or at the delocalized one (hydrogen-like or band states). The wave function of the former is very compact, while of the latter - diffuse, effective mass like. It is associated with a large lattice relaxation effect. Due to a large vibronic barrier while separating these states the impurities exhibit metastability. Exposure to light with energy larger than the photoionization energy of the ground state transforms impurity from the ground (deep) state to the metastable state, which should lead to the change of the refractive index, and thus can be used in efficient writing of a dispersive grating. On the basis of the above considerations we successfully performed continuous wave degenerate four-wave mixing (cw DFWM) experiments on $\mathrm{Cd}_{1-x} \mathrm{Mn}_{x} \mathrm{Te}_{1-y} \mathrm{Se}_{y}$ doped with In crystal (with $x=0.1$ and $y=0.03$ ).

The $\mathrm{Cd}_{1-x} \mathrm{Mn}_{x} \mathrm{Te}_{1-y} \mathrm{Se}_{y}$ doped with In crystal is a diluted magnetic semiconductor. The crystals were grown by the Bridgman method and they were intentionally doped with indium. They are $n$-type with room temperature electron concentration of the order a few times $10^{16} \mathrm{~cm}^{-3}$. The crystal has a direct energy gap of about $1.7 \mathrm{eV}$ [4]. During measurements the crystal was placed in an Oxford CF104 cryostat equipped with a temperature controller. The DFWM experiment was performed at a wavelength of $935 \mathrm{~nm}$ from a Ti:sapphire cw COHERENT model 899 laser. This wavelength coincides with the photoionization spectrum of 
the localized centers in the crystal. The laser beam was split with a beam splitter into two almost equal intensity "write" beams, which travel equal path lengths before crossing at an angle $\Theta$ inside the sample. Their interference forms a sinusoidal pattern which creates a spatial distribution of phototransformed In metastable centers. Due to a difference in a complex dielectric constant when In centers are in the localized and effective mass states, this population distribution acts as an index-of-refraction grating. This grating was probed with the another beam of the same wavelength as the "write" beams, counterpropagating to one of the "write" beams. The intensity of the "read" beam was always kept at least below $10 \%$ of the intensity of one of the "write" beams in order to minimize the contribution to the FWM signal from the grating formed by the "read" beam with one of the "write" beams and to minimize the grating erasure by the "read" beam.

Strong photomemory effect was observed in these samples in the magnetization measurements [4]. Magnetization of the crystals was increasing upon illumination by the near-infrared light, which was ascribed to the formation of bound magnetic polarons formed upon illumination. The effect is metastable at low temperatures and coincident with the persistent photoconductivity effect, simultaneously observed in the CdMnTeSe:In crystals.

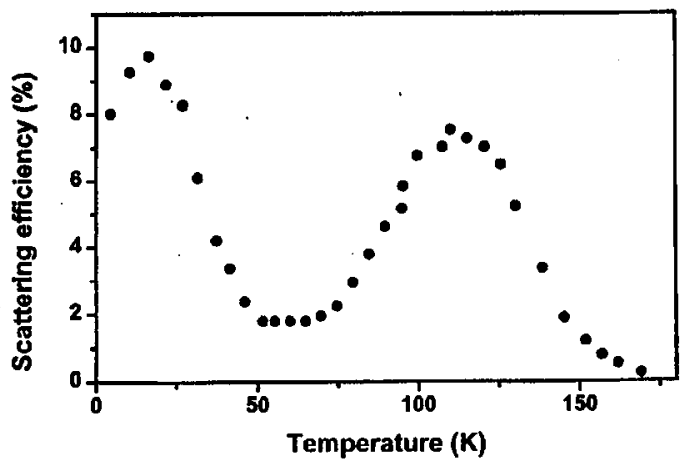

Fig. 1. Temperature dependence of the scattering efficiency of a photoinduced grating in $\mathrm{Cd}_{0.9} \mathrm{Mn}_{0.1} \mathrm{Te}_{0.97} \mathrm{Se}_{0.03}$ :In crystal.

The temperature dependence of the scattering efficiency of the grating photoinduced in this crystal is shown in Fig. 1. The FWM scattering efficiency is above $10 \%$ which means that the local changes of refractive index are responsible for the creation of the light induced grating. The scattering efficiency is temperature dependent due to a spontaneous decay of the grating caused by thermal recovery of the In impurity from the metastable effective mass state to the localized ground state. At low temperature the not equal write beams and stray light coming from scattering on cryostat windows, internal surfaces of the cryostat and sample surfaces cause a complete phototransformation of indium from the localized state to the delocalized one and thus erase the grating.

The temperature dependence of the DFWM scattering efficiency reveals two peaks at temperatures $T_{1}=17 \mathrm{~K}$ and $T_{2}=110 \mathrm{~K}$. These two peaks are associated 
with two types of metastable centers with a different value of energy barrier separating delocalized and localized states. The peak at a temperature $T_{2}=110 \mathrm{~K}$ is associated with the center with an activation energy of $138 \mathrm{meV}$, calculated from the high temperature part of the peak. This value agrees very well with the results of deep level transient spectroscopy (DLTS) [5]. The peak at $T_{1}=17 \mathrm{~K}$ has not been detected in the DLTS experiment. This peak is associated with the center with a lower activation energy of $12 \mathrm{meV}$, approximately.

One of the key physical problems related to such a kind of centers is the question of the number of electrons in the deep state (the delocalized one is the one-electron state), i.e. the sign of the Hubbard correlation energy $U$.

The FWM scattering efficiency is proportional to the square of the difference of the refractive index changes between peaks and valleys of the grating [6]. This value is proportional to the difference in population of effective mass states in the peaks and in the valleys. The last value is given by the solution of the rate equation describing the population of the effective mass and localized states under the equilibrium conditions during illumination. The rate equation for the negative $U$ case are different than for the positive $U$ case due to the two-electron character of the localized state in the $U<0$ case.

The solutions of the rate equations show that the power dependence of the FWM signal for the $U>0$ case is proportional to the square of power of the "write" beams but for the $U<0$ depends on the temperature of the measurements. If the measurements are performed at $T \ll T_{\max }\left(T_{\max }\right.$ is the temperature when the FWM scattering efficiency reaches a maximum value) then the power dependence of the FWM signal is also proportional to the square of "write" beams, but if $T \gg T_{\max }$ then the FWM signal depends linearly on the power of the "write" beams. This experiment may be a convenient test of the sign of the Hubbard energy of the system. Table summarizes the results of the above considerations.

\section{TABLE}

The power dependence of the FWM scattering efficiency for $U>0$ and $U<0$ at different temperatures.

\begin{tabular}{l|c|c}
\hline \hline & $U>0$ & $U<0$ \\
\hline$T \ll T_{\max }$ & $\eta \propto P^{2}$ & $\eta \propto P^{2}$ \\
$T \gg T_{\max }$ & $\eta \propto P^{2}$ & $\eta \propto P$
\end{tabular}

The power dependence of the FWM scattering efficiency in $\mathrm{Cd}_{0.9} \mathrm{Mn}_{0.1} \mathrm{Te}_{0.97} \mathrm{Se}_{0.03}$ : In crystals depends on temperature and it is in agreement with the negative $U$ case for both centers observed in the experiment. Figure 2 shows examples of power dependences of the FWM scattering efficiency for two temperatures: $T \gg T_{\max }$ and $T \ll T_{\max }$ for the center with a higher value of the energy barrier $\left(T_{\max }=110 \mathrm{~K}\right)$. The results for the lower temperature are affected by the second peak at $T_{\max }=17 \mathrm{~K}$. Therefore the exponent of the FWM power 

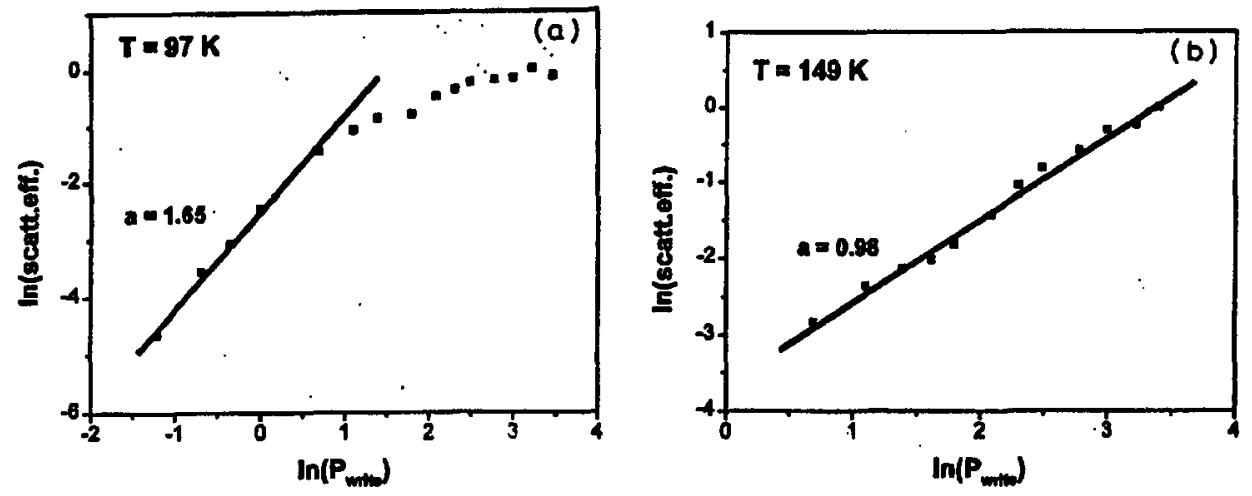

Fig. 2. Temperature dependence of the FWM scattering efficiency on the power of the "write" beams for two temperatures $T=97 \mathrm{~K}(\mathrm{a})$ and $T=149 \mathrm{~K}$ (b).

dependence is lower than 2. These negative $U$ properties of the bistable centers in $\mathrm{Cd}_{0.9} \mathrm{Mn}_{0.1} \mathrm{Te}_{0.97} \mathrm{Se}_{0.03}$ have been also found in the magnetization measurements [4].

In conclusion, we showed that $\mathrm{Cd}_{0.9} \mathrm{Mn}_{0.1} \mathrm{Te}_{0.97} \mathrm{Se}_{0.03}$ crystals doped with indium donors can be efficiently used in writing holographic gratings in the near infrared region. The temperature dependence of the FWM scattering efficiency on the power of the incident "write" beams can show in a quite straightforward way the sign of the Hubbard correlation energy $U$ of the bistable centers in the semiconductors.

\section{Acknowledgments}

This work was partially supported by the grant No. 2 P03B 08709 of the Committee for Scientific Research.

\section{References}

[1] R.A. Linke, T. Thio, J.D. Chadi, G.E. Devlin, Appl. Phys. Lett. 65, 16 (1994); R.L. MacDonald, R.A. Linke, G.E. Devlin, M. Mizuta, Opt. Lett. 20, 1322 (1995).

[2] A.I. Ryskin, A.S. Shcheulin, B. Koziarska, J.M. Langer, A. Suchocki, I.I. Buczinskaya, P.P. Fedorov, B.P. Sobolev, Appl. Phys. Lett. 67, 31 (1995); B. Koziarska, J.M. Langer, A. Suchocki, A.I. Ryskin, A.S. Shcheulin, Acta Phys. Pol. A 88, 1010 (1995).

[3] D.D. Nolte, J. Appl. Phys. 79, 7514 (1996); J.M. McKenna, D.D. Nolte, W. Walukiewicz, P. Becla, Appl. Phys. Lett. 68, 735 (1996).

[4] T. Wojtowicz, S. Koleśnik, I. Miotkowski, J.K. Furdyna, Phys. Rev. Lett. 70, 2317 (1993).

[5] T. Wojtowicz, G. Karczewski, N.G. Semaltianos, S. Koleśnik, I. Miotkowski, M. Dobrowolska, J.K. Furdyna, in: Proc. Int. Conf. on Defects in Semiconductors, Gmunden (Austria) 1993, in series Defects in Semiconductors, Vol. 17, Eds. H. Heindrich, W. Jantsch, Trans. Tech. Publications Ltd., Brookfield, Aedermansdorf 1994, p. 1203.

[6] H. Kogelnik, Bell System Tech. J. 40, 2909 (1969). 